\title{
BMJ Open Safety and feasibility of sublingual microcirculation assessment in the emergency department for civilian and military patients with traumatic haemorrhagic shock: a prospective cohort study
}

To cite: Naumann DN, Mellis C, Smith IM, et al. Safety and feasibility of sublingual microcirculation assessment in the emergency department for civilian and military patients with traumatic haemorrhagic shock: a prospective cohort study. BMJ Open 2016;6: e014162. doi:10.1136/ bmjopen-2016-014162

- Prepublication history for this paper is available online. To view these files please visit the journal online (http://dx.doi.org/10.1136/ bmjopen-2016-014162).

Received 5 September 2016 Revised 14 October 2016 Accepted 5 December 2016

CrossMark

For numbered affiliations see end of article.

Correspondence to Dr Sam D Hutchings; sam. hutchings@nhs.net

\section{ABSTRACT}

Objectives: Sublingual microcirculatory monitoring for traumatic haemorrhagic shock (THS) may predict clinical outcomes better than traditional blood pressure and cardiac output, but is not usually performed until the patient reaches the intensive care unit (ICU), missing earlier data of potential importance. This pilot study assessed for the first time the feasibility and safety of sublingual video-microscopy for THS in the emergency department (ED), and whether it yields useable data for analysis.

Setting: A safety and feasibility assessment was undertaken as part of the prospective observational MICROSHOCK study; sublingual video-microscopy was performed at the UK-led Role 3 medical facility at Camp Bastion, Afghanistan, and in the ED in 3 UK Major Trauma Centres.

Participants: There were 15 casualties (2 military, 13 civilian) who presented with traumatic haemorrhagic shock with a median injury severity score of 26 . The median age was 41 ; the majority $(n=12)$ were male. The most common injury mechanism was road traffic accident.

Primary and secondary outcome measures:

Safety and feasibility were the primary outcomes, as measured by lack of adverse events or clinical interruptions, and successful acquisition and storage of data. The secondary outcome was the quality of acquired video clips according to validated criteria, in order to determine whether useful data could be obtained in this emergency context.

Results: Video-microscopy was successfully performed and stored for analysis for all patients, yielding 161 video clips. There were no adverse events or episodes where clinical management was affected or interrupted. There were 104 (64.6\%) video clips from 14 patients of sufficient quality for analysis.

Conclusions: Early sublingual microcirculatory monitoring in the ED for patients with THS is safe and feasible, even in a deployed military setting, and yields videos of satisfactory quality in a high proportion of
Strengths and limitations of this study

- This study is the first to report sublingual videomicroscopy in the emergency department or in a deployed military environment for patients with traumatic haemorrhagic shock (THS) (ie, before arrival in the intensive care unit).

- Although this study is prospective and multicentred, generalisability may be limited by the low number of patients and their clinical heterogeneity.

- Only safety and feasibility were assessed during this pilot study, and are presented without further analysis of the microcirculatory parameters of recorded video clips.

- Data from this pilot study may help to guide other investigations towards the study of early microcirculatory behaviour following THS.

cases. Further investigations of early microcirculatory behaviour in this context are warranted.

Trial registration number: NCT02111109.

\section{BACKGROUND}

There has been considerable interest in the disruption of the microcirculatory endothelium and endothelial glycocalyx following traumatic haemorrhagic shock (THS). Dysfunctional sublingual microcirculation following THS has been reported to be a good predictor of subsequent organ failure when measured in patients admitted to the intensive care unit (ICU) ${ }^{2}$ The ability to maintain microcirculatory perfusion during early THS has been shown to be associated with more rapid reversal of the shock state during 
resuscitation in a large animal experimental model. ${ }^{3}$ There may be some circumstances where microcirculatory flow does not follow global haemodynamics and parameters such as cardiac output and blood pressure no longer act as reliable surrogate markers for perfusion. ${ }^{4}$ In such circumstances, microcirculatory monitoring may offer more reliable guidance for resuscitation by adding information about true end-organ perfusion. The implications of bedside point-of-care microcirculatory parameters have not yet been realised but may have far-reaching utility in civilian and military contexts.

Although it seems intuitive that microcirculatory readings from earlier time points closer to point of injuryespecially before the definitive cessation of bleeding-may offer diagnostic and prognostic value following major trauma, this has not yet been investigated. Some investigators have performed sublingual microcirculatory assessment in the emergency department (ED) for patients with sepsis ${ }^{5}$ and acute decompensated heart failure, ${ }^{6}$ but this has not yet been performed for trauma patients. It is possible that researchers have not attempted sublingual videomicroscopy for trauma patients in the ED because of the constraints imposed by clinical urgency and environmental uncertainty, lack of capacity to consent, multiple interventions and rapid transfer of the patient. Such a scenario is also likely to be noisy and crowded, with limited space and time at the bedside-conditions that may be even more hostile in the deployed military context. Conversely, the ICU offers a more 'placid' environment with a stationary patient, increased space and time and more stable physiology, even when patients are critically unwell. However, by the time of ICU arrival, patients may have received multiple resuscitative interventions, with unknown impact on the predictive value of sublingual video-microscopy. It is therefore important to establish the feasibility of microcirculatory monitoring within the ED as a basis for studies to determine its clinical utility.

We present for the first time the feasibility of obtaining sublingual video-microscopy video clips during the emergency presentation of patients with THS in the ED. We hypothesised that non-invasive microcirculatory imaging in this emergency context is safe, feasible, does not interfere with clinical management and provides data of sufficient quality for meaningful analysis.

\section{METHODS}

Study design and setting

A prospective observational pilot study was undertaken to assess whether sublingual video-microscopy to image the microcirculation was feasible and safe for civilian and military patients with THS, and whether the captured video clips were of high enough quality for analysis.

\section{Patient selection}

Patients were enrolled into the MICROSHOCK study (ClinicalTrials.gov Identifier: NCT02111109). ${ }^{7}$ Patients were eligible for inclusion if there was evidence of haemorrhagic shock, and all of the following features: (1) injury mechanism consistent with blood loss; (2) the patient is intubated and ventilated; (3) serum lactate concentration $>2 \mathrm{mmol} / \mathrm{L}$; and (4) the patient has received any blood products during initial resuscitation. Patients were recruited as soon as possible after arrival at three UK Major Trauma Centres (Queen Elizabeth Hospital, Birmingham; Kings College Hospital and Royal London Hospital, London). This was either in the ED or ICU. The current study includes the first 13 civilian patients recruited in ED and a further 2 deployed soldiers enrolled in the ED at the Role 3 medical facility in Camp Bastion during the Afghanistan conflict.

\section{Sublingual video-microscopy}

Sublingual microcirculation was visualised in the civilian patients using incident dark field (IDF) video-microscopy (Cytocam, Braedius Medical B.V., Huizen, The Netherlands). Military patients were scanned using a sidestream dark field (SDF) device (MicroVision Medical, Amsterdam, The Netherlands). IDF is a newer technology with higher resolution and larger field of view, but produces comparable results. ${ }^{8}$ The devices are positioned towards the sublingual mucosa and manoeuvred until a clear image of the microcirculation is acquired. Video clips (preferably lasting at least $5 \mathrm{~s}$ each) are then recorded and stored for off-line analysis using dedicated computer software (Automated Vascular Analysis V.3.02, Microvision Medical, The Netherlands). At least 3 (but preferably 5) individual video clips are required for data analysis according to consensus agreement, ${ }^{9}$ but this does not limit the number of videos that can be captured. In this study, as many videos as possible were recorded to ensure a sufficient number of analysis quality. For SDF video images, continuous video was taken rather than short clips; this was later spliced into high-quality segments (each lasting $5 \mathrm{~s}$ ) for computer analysis.

\section{Training}

Sublingual video-microscopy was undertaken by dedicated research clinicians and research nurses who had been trained in the technique by an expert user and the study's chief investigator (SDH) to a standard suitable for clinical research. Training was undertaken paying particular attention to standard quality assessment variables, ${ }^{10}$ including the optimisation of stability, focus and illumination, as well as reducing pressure artefact and ensuring that the field of view contained microcirculatory vessels. The rationale and details of these quality domains have been described in detail elsewhere. ${ }^{11}$ Since all patients in the MICROSHOCK study are intubated, users are trained to access the sublingual area with the endotracheal tube in situ.

\section{Capacity and consent}

Owing to the nature of the injuries sustained and physiological status of patients, capacity to consent was absent. 
The REC-approved consent process for enrolment in the study was guided by the Mental Health Act, UK (2005) and is explained in more detail in the study protocol. ${ }^{7}$ In short, the physician in charge of the care of the patient (Nominated Consultee) agreed on the participation of the patient. A close friend or relative could also be approached if appropriate to act as a Personal Consultee. Ultimately, if the participant regained capacity, they were asked for their permission to retain data already collected.

\section{Data collection}

Patient demographics (age, sex) and injury-related details (mechanism of injury, injury severity score (ISS)) were recorded. Physiological parameters from the prehospital evacuation and ED included lowest systolic blood pressure (SBP), lowest Glasgow Coma Score (GCS) and highest lactate (as a surrogate for perfusion). The number and type of blood products were recorded as a measure of haemorrhagic burden. Details regarding sublingual video-microscopy included timings of video capture, profession of user, mechanism of notification of user, number of video clips stored, total length of video capture and type of consent were also noted.

\section{Outcomes}

The outcomes of interest were: (1) safety (absence of adverse events or interference with clinical management); (2) feasibility (successful acquisition and storage of video clips); and (3) the attainment of videos of high enough quality for meaningful data analysis. Quality assessment was undertaken according to a standardised technique that grades 6 domains for each video (including illumination, duration, focus, content, stability and pressure artefact ${ }^{10}$ by a single assessor (DNN) who was blinded to clinical status of the patient. Each domain was graded as optimal ( 0 points), suboptimal but still useable ( 1 point), or unacceptable and unusable (10 points). If any video clip has a score of 10 in any domain, then the video was deemed unusable.

\section{Minimising potential sources of bias}

All patients who triggered a trauma team activation were screened for inclusion in the study, and a log was kept in order to ensure that risk of selection bias was minimised. The training of all video-microscopists was supervised and regularly assessed by the chief investigator to minimise the risk of interuser heterogeneity. Quality assessment of videos was kept blinded to clinical status of the patient, study site and video-microscopist, so that quality grading was as unbiased and consistent as possible.

\section{RESULTS}

\section{Patient characteristics}

There were 15 patients (13 civilians and 2 military) included in the study. The majority of patients $(12 / 15$, $80 \%$ ) were male; the median age was 41 (IQR 30-55) years. All patients were unconscious and intubated at the time of study enrolment, and recruited into the study with agreement by a Nominated Consultee. There were no cases of subsequent withdrawal of consent from the patient once they regained capacity.

\section{Injury burden and physiology}

The most common injury mechanism was road traffic accident $(n=7)$, followed by crush injury $(n=2)$, fall $(n=2)$, penetrating trauma $(n=1)$ and struck by a train $(n=1)$. One military patient had been injured in an improvised explosive device (IED) blast; the other had been crushed by an armoured vehicle. The median ISS for all patients was 26 (23-34). The median lactate in ED was 4.6 (IQR 2.8-7.9) $\mathrm{mmol} / \mathrm{L}$. The median SBP was 79 (IQR 68-105) $\mathrm{mm} \mathrm{Hg}$, and the median lowest GCS before intubation was 9 (IQR 5-12). Patients in this group received a median of 4 (IQR 1.5-6) units of RBCs, 2 (IQR 0-5) units of fresh-frozen plasma (FFP) and 0 (IQR 0-0.5) units of platelets within the first 24 hours. The military patient injured by the IED received 32 units of RBCs, 31 units of FFP and 5 units of platelets.

\section{Video-microscopy}

The IDF device was used for 13 civilian patients, and the SDF device was used for the 2 military patients. Figure 1 illustrates a flow diagram of microcirculatory video acquisition. Video-microscopy was performed by a doctor for 12 patients and nurse for 3 patients. On all occasions, these healthcare professionals were alerted to the arrival of the patient by phone call from the relevant ED. Video-microscopy was performed a median of 80 (IQR 58-138) min after arrival of the patient at the hospital. Where a CT was performed as part of trauma management, this preceded sublingual video-microscopy in all instances.

\section{Safety and feasibility}

Video-microscopy was successfully performed and videos stored for analysis for all patients enrolled in ED. One hundred and sixty-one video clips were stored for analysis, including 151 from civilian patients and 10 from military patients (the long continuous videos acquired for the military patients were spliced into 5 clips each). The median time at the bedside for video capture was 6 (IQR 5-8) min. There were no adverse events, and no incidents reported where clinical management was affected or patient care interrupted.

\section{Quality assessment of videos}

Of all videos retained for analysis, 104 of 161 (64.6\%) were of suitable quality for computer analysis. These videos were acquired from 14 of the patients, with 1 patient having no useable data. A median of 6 (IQR 5-10) video clips per patient were eligible for analysis, exceeding the 3-5 clips recommended by consensus guidance. ${ }^{9}$ The median quality assessment score for 


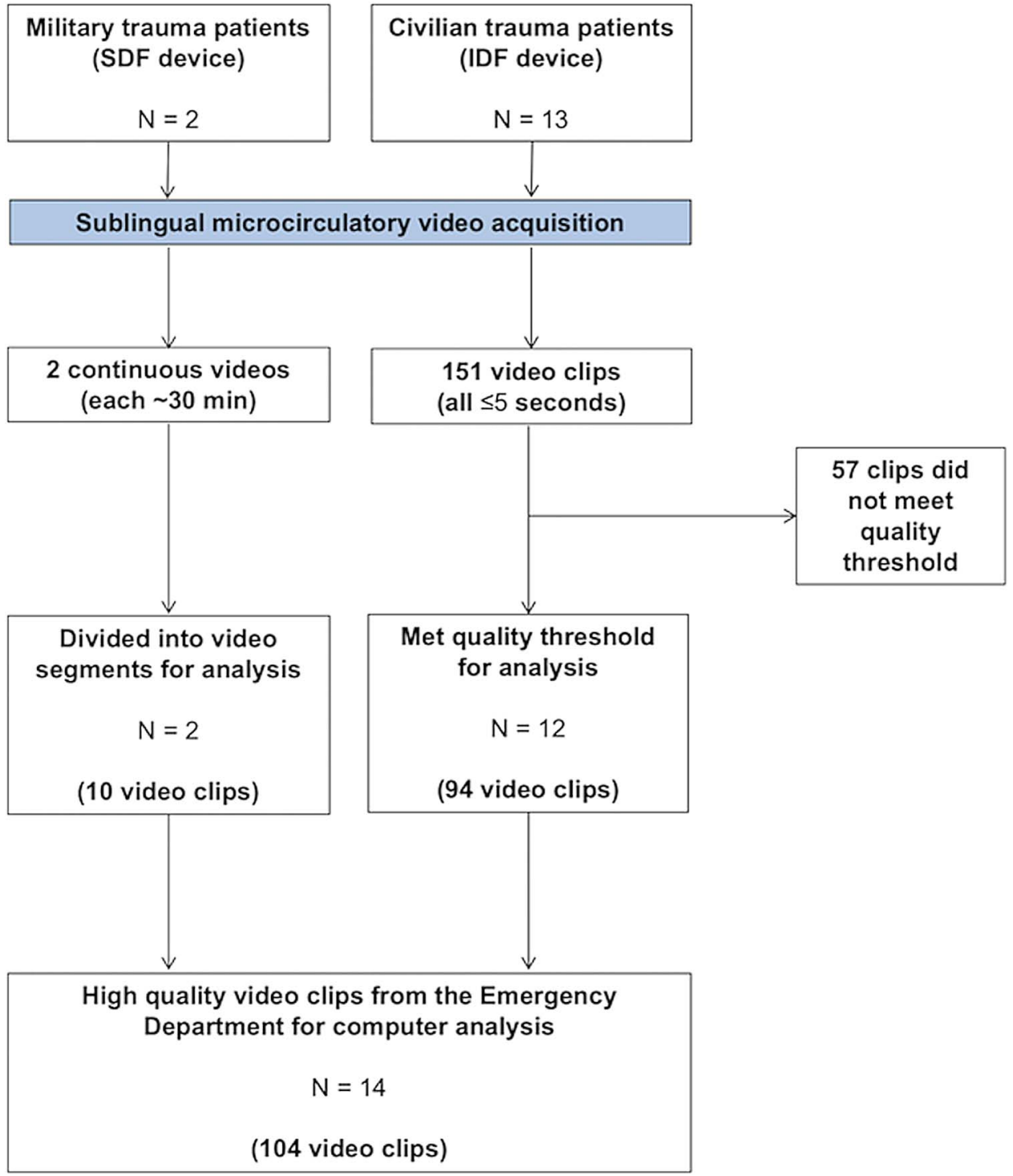

Figure 1 Flow diagram of microcirculatory video clip acquisition for computer analysis. $\mathrm{N}$ indicates the number of study participants at each stage.

useable videos was 2 (IQR 1-2). Of the 57 video clips that were unusable, 18 failed quality assessment on more than 1 domain. The remaining 39 video clips that failed due to a single quality domain included content $(n=14)$, pressure $(n=13)$, stability $(n=6)$, illumination $(n=3)$, focus $(n=2)$ and duration $(n=1)$.

\section{DISCUSSION}

The main finding from this study is that early sublingual microcirculatory monitoring in the ED is feasible and safe for patients with THS, and yields videos that can be used for analysis. Investigation of patients with THS can be performed using this technique without apprehension of interference in clinical management or detriment to the patient. Such non-invasive scanning modalities are commonplace during trauma resuscitation when they are considered to add valuable information, including focused assessment with sonography for trauma, and ultrasound to guide fluid therapy. ${ }^{12}$ Associated training and ongoing validation would be essential components if this technique were to be used in clinical practice.

Patients in this study had a considerable injury burden, with additional haemodynamic compromise according to their physiological and biochemical parameters. Sublingual microcirculatory monitoring was still feasible in this context within the very first hours of their arrival in hospital. Although the clinical utility of such readings is yet to be realised, it is possible that the availability of additional data relating to tissue perfusion may be of value in the resuscitation of such patients. Point-of-care microcirculatory monitoring is not currently used in clinical practice, but innovations to move this technique from research to the clinical domain have been proposed by our group $^{13}$ and others. ${ }^{14}$ If 
point-of-care microcirculatory monitoring is deemed to be a useful resuscitation end point, then it would be important to obtain readings before, during and after interventions, so that changes might be recorded. The current study did not use such methodology, but further investigations into the utility of this technique are warranted.

\section{Obstacles and limitations}

There are known obstacles in the acquisition of early microcirculatory data, which were confirmed in this feasibility study. Patients with THS are critically unwell, and their treatment is urgent and needs to progress uninterrupted. Transfers to radiology, ICU or operating theatre cannot be paused for data acquisition without strong justification. Sublingual video-microscopy has potential to overcome some of these limitations because it is mobile and can follow the patient. We report that it takes a matter of minutes to undertake, and that there was a point in the patient pathway in all cases before patient transfer during which opportunistic videomicroscopy was suitable. In all occasions where crosssectional imaging was undertaken, video-microscopy was performed afterwards. The study investigators did not wish to interfere with the preparation or transfer of patients who needed urgent imaging. If the technique is found to have clinical utility, then there may be some justification in obtaining even earlier readings, and incorporating the technique into the resuscitative pathway.

Although feasibility has been demonstrated, one patient had no videos clips of high enough quality for assessment. Time constraints and interference with video acquisition may increase the risk of such occurrences, and would require continued education, training and maintenance of appropriate skills for data capture in less than ideal (and sometimes adverse) circumstances. User-dependency is a common feature of scanning modalities. Clinical judgement continues to be the optimal management strategy for these emergency scenarios with or without the additional data that microcirculatory monitoring might yield. There were only two military patients included in this study, and the authors acknowledge that firm conclusions cannot be made with these limited data. Further validation is required in such an environment.

The majority of sublingual microcirculatory monitoring is conducted in the research domain, and early bedside point-of-care monitoring of the microcirculation for patients with THS has not been reported. Although limited by a small number of patients, the current study adds to the growing body of evidence that may justify and facilitate the transition of microcirculatory monitoring from research into clinical practice. Restoration of tissue perfusion by directing fluid and inotropic resuscitation towards microcirculatory targets appears to be a viable technique, but is yet to be tested. Some investigators have proposed that plasma may improve microcirculatory function due to its restorative properties. ${ }^{15}$ Detection of microcirculatory dysfunction may have a role in guiding the choice or volume of fluids. Since acquisition of early microcirculatory data is feasible, it is timely to design and implement appropriate studies to examine whether microcirculatory goaldirected therapy is of benefit to patients.

Author affiliations

${ }^{1}$ NIHR Surgical Reconstruction and Microbiology Research Centre, Queen

Elizabeth Hospital, Birmingham, UK

${ }^{2}$ University Hospitals Birmingham NHS Foundation Trust, Queen Elizabeth Hospital, Birmingham, UK

${ }^{3}$ Royal Centre for Defence Medicine, Queen Elizabeth Hospital, Birmingham, UK

${ }^{4}$ Kings College Hospital, London, UK

${ }^{5}$ Queen Elizabeth University Hospital, Govan, Glasgow, UK

${ }^{6}$ Barts Health NHS Trust and Queen Mary University of London, London, UK

${ }^{7}$ Rural Clinical School, University of Queensland, Bundaberg Hospital,

Bundaberg, Queensland, Australia

Acknowledgements The authors wish to thank the research nursing and administrative staff at Kings College Hospital, London; Royal London Hospital, London; and the NIHR Surgical Reconstruction and Microbiology Research Centre, Birmingham.

Contributors SDH conceived, designed and developed the MICROSHOCK study. DNN, MJM and TH contributed to study design modification and protocol amendments. Video acquisition for military patients was undertaken by MJM in Afghanistan. The remainder were performed by DNN and MJM (Birmingham), JM, IMS and TH (Royal London) and SDH and CM (Kings College London). IMS implemented the military study in Birmingham. DNN wrote the manuscript, and all other authors contributed to the development, revision and final version.

Funding The MICROSHOCK study has been funded by the Research Directorate at the Royal Centre for Defence Medicine, as well as the National Institute of Academic Anaesthesia (grant number WKR0-2014-0050) and the National Institute for Health Research. Open access funding is provided by the University of Birmingham.

Competing interests None declared.

\section{Patient consent Obtained.}

Ethics approval NRES Committee Yorkshire \& The Humber-Leeds West and and the civilian Research Ethics Committee (REC Ref 14/YH/0078) and Ministry of Defence Research Ethics Committee (MODREC Ref PPE 281/12) approvals were granted before the start of the study.

Provenance and peer review Not commissioned; externally peer reviewed.

Data sharing statement No additional data are available.

Open Access This is an Open Access article distributed in accordance with the Creative Commons Attribution Non Commercial (CC BY-NC 4.0) license, which permits others to distribute, remix, adapt, build upon this work noncommercially, and license their derivative works on different terms, provided the original work is properly cited and the use is non-commercial. See: http:// creativecommons.org/licenses/by-nc/4.0/

\section{REFERENCES}

1. Chignalia AZ, Yetimakman F, Christiaans SC, et al. The glycocalyx and trauma: a review. Shock 2016;45:338-48.

2. Tachon G, Harrois A, Tanaka S, et al. Microcirculatory alterations in traumatic hemorrhagic shock. Crit Care Med 2014;42:1433-41.

3. Hutchings SD, Naumann DN, Watts S, et al. Microcirculatory perfusion shows wide inter-individual variation and is important in determining shock reversal during resuscitation in a porcine experimental model of complex traumatic hemorrhagic shock. Intensive Care Med Exp 2016;4:17. 
4. Ince $\mathrm{C}$. Hemodynamic coherence and the rationale for monitoring the microcirculation. Crit Care 2015;19(Suppl 3):S8.

5. Trzeciak S, Dellinger RP, Parrillo JE, et al. Early microcirculatory perfusion derangements in patients with severe sepsis and septic shock: relationship to hemodynamics, oxygen transport, and survival. Ann Emerg Med 2007;49:88-98, 98.e1-2.

6. Hogan CJ, Ward KR, Franzen DS, et al. Sublingual tissue perfusion improves during emergency treatment of acute decompensated heart failure. Am J Emerg Med 2012;30:872-80.

7. Hutchings S, Naumann DN, Harris T, et al. Observational study of the effects of traumatic injury, haemorrhagic shock and resuscitation on the microcirculation: a protocol for the MICROSHOCK study. BMJ Open 2016;6:e010893.

8. Hutchings S, Watts S, Kirkman E. The Cytocam video microscope. A new method for visualising the microcirculation using Incident Dark Field technology. Clin Hemorheol Microcirc 2016;62:261-71.

9. De Backer D, Hollenberg S, Boerma C, et al. How to evaluate the microcirculation: report of a round table conference. Crit Care 2007;11:R101.
10. Massey MJ, Larochelle E, Najarro G, et al. The microcirculation image quality score: development and preliminary evaluation of a proposed approach to grading quality of image acquisition for bedside videomicroscopy. J Crit Care 2013;28:913-17.

11. Massey MJ, Shapiro NI. A guide to human in vivo microcirculatory flow image analysis. Crit Care 2016;20:35.

12. Ferrada $P$, Evans $D$, Wolfe $L$, et al. Findings of a randomized controlled trial using limited transthoracic echocardiogram (LTTE) as a hemodynamic monitoring tool in the trauma bay. $J$ Trauma Acute Care Surg 2014;76:31-7; discussion 7-8.

13. Naumann DN, Mellis C, Husheer SL, et al. Real-time point of care microcirculatory assessment of shock: design, rationale and application of the point of care microcirculation (POEM) tool. Crit Care 2016;20:310.

14. Arnold RC, Parrillo JE, Phillip Dellinger R, et al. Point-of-care assessment of microvascular blood flow in critically ill patients. Intensive Care Med 2009;35:1761-6.

15. Tuma M, Canestrini S, Alwahab Z, et al. Trauma and endothelial glycocalyx: the microcirculation helmet? Shock 2016;46:352-7. 